\title{
REDUCED ORDER MODELING OF REACTING SHEAR FLOW
}

\author{
Daehyun Wee ${ }^{\dagger}$, Sungbae Park ${ }^{\dagger}$, Tongxun $\mathrm{Yi}^{\dagger}$, Anuradha Annaswamy ${ }^{\ddagger}$ and Ahmed Ghoniem ${ }^{\S}$ \\ Department of Mechanical Engineering \\ Massachusetts Institute of Technology
}

\begin{abstract}
$\underline{\text { Abstract }}$
Thermoacoustic instability in premixed combustors occurs occasionally at multiple frequencies, especially in configurations where flames are stabilized on separating shear layers that form downstream of sudden expansions or bluff bodies. While some of these frequencies are related to the acoustic field, others appear to be related to shear flow instability phenomena. It is shown in this paper that shear flows can support self-sustained instabilities if they possess absolutely unstable modes. The associated frequencies are predicted using mean velocity profiles that resemble those observed in separating flows and for profiles obtained from numerical simulations, and are shown to match those derived from experimental and numerical investigations. It is also shown that the presence of density profiles compatible with premixed combustion can affect this frequency and can change the absolute instability mode into a convectively unstable mode thereby reducing the possibility of the generation of self-sustained oscillations. A qualitative prediction of the pressure amplitudes resulting from these shear layer modes is shown to be consistent with experimental measurements. The results from the stability analysis are combined with those using the Proper Orthogonal Decomposition (POD) method to yield a reduced-order model.
\end{abstract}

\section{Introduction}

Thermoacoustic instability, which manifests as growing pressure oscillations, is predominantly due to destabilizing interactions between acoustics and heatrelease rate perturbations. Characterized by a positive Rayleigh Index, obtained when the pressure and heat release perturbations are in phase, the instability occurs normally at frequencies that are associated with acoustic modes that can be identified as a longitudinal,

Copyright $@ 2002$ by D. Wee, S. Park, T. Yi,

A.M. Annaswamy and A.F. Ghoniem.

Published by the American Institute of Aeronautics and

Astronautics, Inc. with permission.

\footnotetext{
${ }^{\dagger}$ Graduate research assistant.

$\ddagger$ Principal Research Scientist and Lecturer.

$\S$ Professor, Associate fellow, AIAA.
}

azimuthal, or bulk mode depending on the configuration of the combustor. These interactions have been modeled in a number of recent investigations by including acoustics and heat-release dynamics as primary components [1-7].

In some of the combustor configurations, however, the combustion instability occurs at multiple frequencies, not all of which can be identified with acoustics. In these combustors, a "hydrodynamic" instability has been argued to be the source of the oscillation [8-12]. In particular, the phenomenon of vortex shedding has been observed in combustors equipped with sudden expansions, bluff-bodies, swirlers, etc., all of which are utilized for flame stabilization in premixed systems. A representative configuration of these separating flows is a backward-facing step or dump combustor that has been used extensively in experimental and numerical studies, both for non reacting as well as reacting flows [13-16]. The pressure power spectra measured in these experiments often exhibit multiple peaks, some of which are easily related to existing acoustic modes, but others are not. This raises the possibility that other "resonance" phenomena play a role in determining the overall instability characteristics. Whether the resonances form due to acoustic forcing or through other mechanisms has not yet been proven. The existence of vortices in these cases, which raises the possibility that shear layer dynamics may be involved in the resonance, is confirmed by visualization studies that show flames wrapping around large vortical structures. As summarized by Najm et al. [17], the frequency of the most active oscillations in non reacting flows, or the corresponding vortex shedding frequency, satisfies, in most cases, the criterion $0.05<\mathrm{St}<0.1$, where the Strouhal number is based on the step height and the mean upstream flow speed. Numerical calculations of the same flow, although they are mostly limited to relatively low Reynolds numbers, reproduce the same result. The question that arises is whether self-sustained oscillations produced by a hydrodynamic instability can cause the heat release to be perturbed and as a result feed energy into the acoustic field, thereby producing additional resonant frequencies.

As a representative example of non reacting and reacting flow data, the pressure power spectrum in the experiment of Cohen et al. [13] shows three peaks at 48, 96 and $124 \mathrm{~Hz}$ in the reacting flow, and a dominant peak close to $100 \mathrm{~Hz}$ in the non reacting flow. Solutions, 
analytical in 1D and numerical in 2D, of the acoustic wave equation predict the presence of the modes at 48 $\mathrm{Hz}$ and the $124 \mathrm{~Hz}$, as the quarter mode and threequarter modes of the system, but not the mode at $96 \mathrm{~Hz}$. Moreover the experimental data show that while these two modes appear as broad peaks, the $96 \mathrm{~Hz}$ peak is significantly sharper, implying that the latter is generated perhaps by a different mechanism. It is interesting to note that the $96 \mathrm{~Hz}$ mode scales to a Strouhal number of 0.092 , indicating that its origin may be related to hydrodynamic instability.

In this paper, an attempt is made towards deriving a reduced-order model of reacting shear flow in a combustor with a backward-facing step. By using linear stability theory of shear flow and applying it to separating flows, it is explored whether these flows can support self-sustained oscillations similar to those observed in experimental studies. These results are suitably combined with a POD-based analysis to derive a reduced-order model. In section II, the basic linear stability theory of shear flows is introduced. Using the Green's function for perturbed Euler equation and asymptotic evaluation of integrals, the frequency and the growth rate of the hydrodynamic mode are calculated, and the conditions under which the frequency corresponds to the absolutely unstable mode are delineated. Numerical simulations are used to obtain the mean velocity profiles, and the results from the linear stability analysis are compared with the numerical results. In section III, qualitative predictions of the pressure amplitudes, which may be expected at the shear layer modes, are compared with experimental data. Finally, in section IV, using numerical data, a reduced order model is constructed for potential use in active control design.

\section{Stability Analysis of Separating Shear Flow}

In this section, the separating flow downstream of a backward-facing step is analyzed using linear stability analysis. The objective of the analysis is to determine conditions under which the shear flow can support selfsustained oscillations arising from intrinsic instabilities, even in the absence of upstream perturbations or resonance with other oscillations such as those due to acoustics. These forms of instabilities are known as absolutely unstable modes and have been shown to arise in a number of shear flows [18, 19].

This section is organized as follows. In section II.1, basic formulation of linear stability analysis is described. In section II.2, the definition of absolutely unstable modes and conditions under which they may be expected are presented. In section II.3, the effect of flow parameters including the local shear layer thickness and the magnitude of the backflow on the local stability properties is examined, and how these local phenomena may be used to predict global properties is explained. Both the unstable frequency and the location of the strongest unsteadiness are compared with the predictions obtained from a numerical investigation of the unsteady flow. In section II.4, the analysis is extended to the case with finite density gradients across the layer to examine the impact of combustion of the absolute stability properties of the flow.

\section{II.1 Formulation of Local Stability Analysis}

Assuming inviscid and incompressible channel flow, the 2-D Euler equation and continuity equations are combined to obtain the governing 2-D vorticity equation [18]

$$
\left[\frac{\partial}{\partial t}+\frac{\partial \Psi}{\partial y} \frac{\partial}{\partial x}-\frac{\partial \Psi}{\partial x} \frac{\partial}{\partial y}\right] \nabla^{2} \Psi=u_{s}(t) \delta(x) \delta(y)
$$

where $\Psi$ is the stream function, the vorticity is $\Omega \vec{k}=\nabla \times \vec{V}=-\nabla^{2} \Psi \vec{k}$, and $\vec{V}=(u, v)=\left(\frac{\partial \Psi}{\partial y},-\frac{\partial \Psi}{\partial x}\right)$ is the velocity in the $x$ and $y$ direction, respectively, $\delta$ is the Dirac delta function, and $\vec{k}$ is the unit vector in the $z$ direction. The source term $u_{s}(t) \delta(x) \delta(y)$ represents the effect of an upstream velocity perturbation at the backward-facing step. Also, we assume that the mean flow field, $U$, depends only on $y$, which implies that the stability analysis is 'local'. Linearizing Eq. (1), we obtain the following equation for the perturbation

$$
\nabla^{2} \psi_{t}+U \cdot \nabla^{2} \psi_{x}-U^{\prime \prime} \psi_{x}=u_{s}(t) \delta(y)
$$

The boundary condition for this equation are derived from the impermeability condition at the upper and lower walls as

$$
\psi(a)=\psi(b)=0, a \leq y \leq b .
$$

The Green's function, $G$, is obtained from the impulse response of the flow as

$$
\left[\frac{\partial}{\partial t}+U(y) \frac{\partial}{\partial x}\right] \nabla^{2} G-U^{\prime \prime}(y) \frac{\partial G}{\partial x}=\delta(t) \delta(y) .
$$

Taking Fourier transforms in $x$ and $t$, we obtain

$$
\hat{\hat{G}}^{\prime \prime}-\alpha^{2} \hat{\hat{G}}-\frac{U^{\prime \prime}}{U-c} \hat{\hat{G}}=\frac{\delta(y)}{i \alpha(U-c)} \text {. }
$$

where $\hat{\hat{G}}$ and $G$ are related according to

$$
\begin{aligned}
& G(x, y, t)=\frac{1}{2 \pi} \int_{F} \hat{G}(\alpha, y, t) e^{i \alpha x} d \alpha, \\
& \hat{G}(x, y, t)=\frac{1}{2 \pi} \int_{L} \hat{\hat{G}}(\alpha, y, \omega) e^{-i \omega t} d \omega .
\end{aligned}
$$

$\alpha$ and $\omega$ are the wave number and the frequency respectively, and $c=\omega / \alpha$. To meet the causality condition, the path of integration $L$ should be placed over all singularities in $\omega$ plane, and the path $F$ should be the real axis in the $\alpha$ plane. 


\section{II.2 Identification of an Absolute Mode}

It should be noted that the objective of the analysis is to examine the long-term behavior of the flow near the step, i.e. at $x=0$. The mode that dominates the flow along this line is defined as the absolute mode of the flow. If this mode is unstable, the flow is absolutely unstable, and it exhibits growing waves that are not convected out from the observation point, i.e. oscillations may persist and the flow demonstrates the properties of an unstable oscillator. For unstable but absolutely stable flows, the input is amplified downstream and the growing waves are convected out. These flows are referred to as convectively unstable flows. The major difference between absolute and convective instabilities is that in the first case, oscillations persist at a frequency determined by the properties of the flow, i.e. the flow achieves self resonance, while in the second external forcing or resonance with other oscillators must occur before overall sustained oscillations can be observed. Following the method used in [18], we now derive the frequency and the growth rate selection criterion for absolute modes.

The homogeneous part of Eq. (5) is the well-known Rayleigh equation ${ }^{1}$ whose solutions are denoted as $\eta_{1}(y)$ and $\eta_{2}(y)$ and satisfy the boundary conditions that $\eta_{1}(a)=0$ and $\eta_{2}(b)=0$. The forced response $G$ of Eq. (4) can be determined using $\hat{\hat{G}}$, which is the solution of (5), and the integrals in (6) and (7). $\hat{\hat{G}}$ in turn, can be computed as

$$
\hat{\hat{G}}(y)=\int_{y^{\prime}=a}^{b} \frac{H\left(y, y^{\prime}\right)}{W\left(y^{\prime}\right)} \delta\left(y^{\prime}\right) d y^{\prime}=\frac{H(y, 0)}{W(0)}
$$

where

$$
H\left(y, y^{\prime}\right)= \begin{cases}\frac{\eta_{1}(y) \eta_{2}\left(y^{\prime}\right)}{i \alpha\left(U\left(y^{\prime}\right)-c\right)} & y<y^{\prime} \\ \frac{\eta_{1}\left(y^{\prime}\right) \eta_{2}(y)}{i \alpha\left(U\left(y^{\prime}\right)-c\right)} & y^{\prime}<y\end{cases}
$$

and $W(y)$ is the Wronskian $W(0)=\eta_{1} \eta_{2}^{\prime}-\left.\eta_{1}^{\prime} \eta_{2}\right|_{y=0}$ which is non-zero when $\eta_{1}$ and $\eta_{2}$ are two linearly independent solutions. To guarantee a nontrivial solution for the homogeneous equation which satisfies both boundary conditions, $\eta_{1}$ and $\eta_{2}$ must also be the eigenfunction $\eta_{\text {eigen }}$, and satisfy $W=0$, which is the

${ }^{1}$ The standard form for the Rayleigh equation is given by $\eta_{y y}-\left(\alpha^{2}+\frac{U^{\prime \prime}}{U-c}\right) \eta=0$ and $\eta(a)=\eta(b)=0$,

where $\eta(y)$ is the mode shape $[20,21]$. same as the dispersion relation for the Rayleigh equation.

To obtain $G$, we examine Eqs. (6) and (7). The inverse transform in (7), from the frequency to the time domain, is performed by adding the residues of singularities in the integrand $\hat{\hat{G}}$. This is obtained as

$$
\hat{G}=-i \frac{\left.H(y, 0)\right|_{\omega=\omega_{1}(\alpha)}}{\left.\frac{\partial W}{\partial \omega}\right|_{\omega=\omega_{1}(\alpha)}} e^{-i \omega_{1} t}
$$

where $\omega_{j}$ is the eigen-frequency of the homogeneous solution corresponding $\alpha$ with $W(\omega)=0$, and $\omega_{1}$ corresponds to the most unstable branch of the eigenfrequency. That is, $\omega_{1}=\arg \left[\max _{\omega}\left(\omega_{i}\right)\right]$. Note that $H(y, 0)$ is now defined on the singularity, $W=0$, and is given by

$$
H(y, 0)=\frac{\eta_{\text {eigen }}(y) \eta_{\text {eigen }}(0)}{i \alpha(U(0)-c)} .
$$

The long-time asymptotic solution of $G$ can be evaluated by applying the method of steepest descent path to Eq. (6). It can be shown that the behavior of $G$ depends only on one mode for each line $x / t=$ const. Especially, on the line $x=0$, the large-time asymptotic behavior of $G$ is shown to be dependent only on one mode, $\left(\omega_{0}, \alpha_{0}\right)$, where $\omega_{0}=\omega_{1}\left(\alpha_{0}\right)$ and $\alpha_{0}$ is such that

$$
\frac{d \omega_{1}}{d \alpha}\left(\alpha_{0}\right)=0
$$

Thus this mode determines the frequency and the stability of the oscillation on the line $x=0$ for large $t$, and hence corresponds to an absolute mode. Equation (11) shows that the group velocity of the absolute mode is zero, which seems to be physically reasonable, because all other modes with non-zero group velocity are swept out from the point, eventually. The shear layer forms a locally unstable oscillator, only when it is absolutely unstable. That is, when

$$
\omega_{0, i}>0 \text {. }
$$

Given the dominance of the absolute mode, $G$ in (6) is affected mostly near $\alpha=\alpha_{0}$ and hence

$$
\begin{aligned}
G & \cong \frac{1}{2 \pi} \int \hat{G} e^{i \alpha x} \delta\left(\alpha-\alpha_{0}\right) d \alpha \\
& =-\frac{1}{2 \pi} \frac{i[H(y, 0)]_{\alpha_{0}, \omega_{0}}}{\left(\frac{\partial W}{\partial \omega}\right)_{\alpha_{0}, \omega_{0}}} \times e^{i\left(\alpha_{0} x-\omega_{0} t\right)}
\end{aligned}
$$




$$
\bar{G}(x, y, s)=-\frac{i}{2 \pi} \frac{[H(y, 0)]_{\alpha_{0}, \omega_{0}} e^{i \alpha_{0} x}}{\left(\frac{\partial W}{\partial \omega}\right)_{\alpha_{0}, \omega_{0}}} \cdot \frac{1}{s+i \omega_{0}}
$$

Noting that $\omega_{0}$ is complex, it follows that $\bar{G}$ is a second order oscillator, which is unstable if Eq. (12) is satisfied. The time-domain representation of the shearlayer oscillation may be derived from (14) as

$$
\left[\frac{d^{2}}{d t^{2}}-2 \omega_{0, i} \frac{d}{d t}+\left|\omega_{0}\right|^{2}\right] \eta_{s}(t)=\left[\frac{d}{d t}-i \omega_{0}^{*}\right] u^{\prime}\left(x_{\text {seep }}, t\right)
$$

where $\psi(x, y, t)=\psi_{s}(x, y) \eta_{s}(t), \psi_{s}(x, y)$ is the spatial pattern of the mode, and $\eta_{s}(t)$ is the temporal component of the mode.

\section{II.3 Absolute Modes for Separating Flow}

In section II.2, we considered the shear layer dynamics in a channel with impermeable walls, and showed that the dynamics are that of a second-order oscillator with a frequency $\omega_{0}$ and a wave number $\alpha_{0} \cdot \omega_{0}$ and $\alpha_{0}$ (i) satisfy the dispersion relation $W=0$, (ii) satisfy Eq. (11) since they correspond to the absolute mode, and (iii) satisfy Eq. (12) if this absolute mode is unstable. In this section, the properties (i)-(iii) are discussed in the context of a separating shear flow downstream of a backward-facing step. The complex frequency of the absolute mode, determined by Eq. (11), is evaluated using the cusp-map method [21, 22]. The following velocity profile is used to model a separating flow downstream a backward facing step;

$$
\begin{gathered}
U(y)=\frac{\beta-1}{2}+\frac{\beta+1}{2} \tanh \left(\frac{y}{\delta}\right)-\beta \tanh \left(\frac{y-a}{\delta_{w}}\right) \\
-\tanh \left(\frac{y-b}{\delta_{w}}\right) \quad(-a \leq y \leq b)
\end{gathered}
$$

where $\beta$ is the ratio of backflow to the forward flow, $\delta$ defines the shear layer thickness, and $\delta_{w}$ is the boundary layer thickness at the wall. The third and fourth terms are added to mimic the impact of the nonslip boundary condition. When $\delta$ and $\delta_{w}$ are reasonably small, the profile approximates the mean velocity distribution of step flows reasonably well. Figure 1 shows the mean velocity profile given by Eq. (16) for selected values of the parameters. We assume $a=-1, b=1$, and $\delta_{w}=0.1$, where all length scales are normalized with respect to the step height. These values are chosen to simulate the case with expansion ratio 2 . We note that realistic values of $\beta$ vary from 0.3 to 0.4 for the mean velocity profile in a backward-facing step flow at high Reynolds number. ${ }^{2}$ We also note that in a

\footnotetext{
${ }^{2}$ See, for example, [14, 17, 23, 24].
}

typical recirculating flow, both the shear layer thickness and the backflow changes downstream of the step. The profile in Eq. (16), however, is intended to model the velocity distribution at any particular section, using the original assumption that the flow is parallel.

The absolute mode frequencies supported by these velocity profiles were calculated for various values of $\delta$ and $\beta$, and the results are depicted in Figure 2. Because the Strouhal number is often based on the mean upstream velocity, $U_{0}$, we normalize the value of $\omega_{0}$ as $\omega_{0} / U_{0}$, where $U_{0}=\int_{y=-1}^{1} U(y) d y .^{3}$ Results show that for a fixed value of $\delta$, the growth rate of the unstable mode switches from being convectively unstable to absolutely unstable as the backflow increases. Moreover, the growth rate of the absolutely unstable mode increases with increasing $\beta$. Similar observation can be made for $\delta$; for a fixed backflow, decreasing $\delta$ can lead to an absolutely unstable flow, with further drop in $\delta$ causing an increase in the absolute mode growth rate. We also note that the mode frequency, $\omega_{0, r}$, is not strongly dependent on $\beta$, especially for larger values of $\delta$, i.e. changes in the backflow, which is observed within the recirculation zone of a step flow, does not impact the frequency of the absolutely unstable mode significantly; they impact, however, the growth rate and hence absolute modes are more likely to originate at the section with strongest backflow. Because the shear layer thickness increases downstream of the step, reaching almost at the same order as the step height in the middle of the recirculation zone, we focus on the case with $\delta \approx 0.4$. This case corresponds to a shear layer thickness of beta $=0.3$, delta $_{w}=0.1$

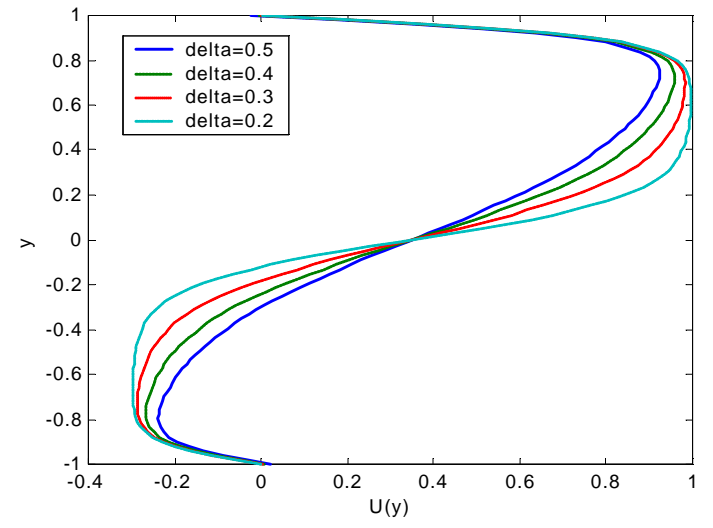

Figure 1 Mean velocity profile given by Eq. (16) for different parameters.

\footnotetext{
${ }^{3}$ Because $U(y)$ is already a nondimensional velocity profile, this does not violate the dimension matching principle.
}

4 
almost unity, i.e. on the order of magnitude of the step height (see fig 1 ). As shown in Figure $2, \omega_{0, r} \approx 0.6$ at $\delta \approx 0.4$, and the corresponding frequency of the absolute mode scales to $\mathrm{St}=\omega_{0, r} / 2 \pi \cong 0.1$ for a range of $\beta$. This may explain the observation that strong fluctuations in backward-facing step flows occurs roughly near $\mathrm{St}=\frac{f h}{U_{0}} \cong 0.1$, where $h$ is the step height and $U_{0}$ is the upstream mean velocity of the flow.

Using these results, we can classify the stability characteristics of the mean velocity profiles at each section of backward-facing step flows (see Figure 3 ). The objective of this classification is to determine the most likely origin of the most-unstable absolute mode within the recirculation zone, and its overall impact on the flow. At locations near the step, the shear layer is very thin, and the backflow is weak, and hence, according to Figure 2, the flow is absolutely stable. Near the middle of the recirculation zone, the mean velocity profile shows strong back flow, and the shear layer thickness is comparable to the step height; and hence the flow may become absolutely unstable at a frequency near $\mathrm{St} \cong 0.1$. Note that, from previous observations, the frequency is only weakly dependent on the backflow and thus the absolute instability will be supported at the section of maximum backflow. Towards the end of the recirculation zone, the shear layer thickness remains almost the same, because the flow is bounded by the upper and lower walls, while the backflow diminishes. Figure 2 shows that under these conditions, the frequency should remain the same, $\mathrm{St} \cong 0.1$, while the flow becomes absolutely stable.

The following global picture of a separating shear layer instability, which is supported by strong backflow, emerges form the previous analysis. Selfsustained oscillation occurring in the middle of the recirculation zone, where the backflow is strongest, propagates upstream and downstream. The frequency of these oscillations is determined by the thickness of the shear layer at the point of the initiation, i.e. the middle of the recirculation zone. The evidence from experimental measurements and numerical simulations is that the thickness of the shear zone around the middle of the recirculation zone is of the order of magnitude of the step height. Oscillations associated with an absolutely unstable mode grow in place while spreading upstream and downstream. Once these oscillations reach an absolutely stable but convectively unstable region, the flow becomes convectively unstable, i.e. oscillation at the local convective mode with the most unstable frequency may be excited. While the growth of these oscillations, associated with the convective mode at the step, are swept by convection downstream, those

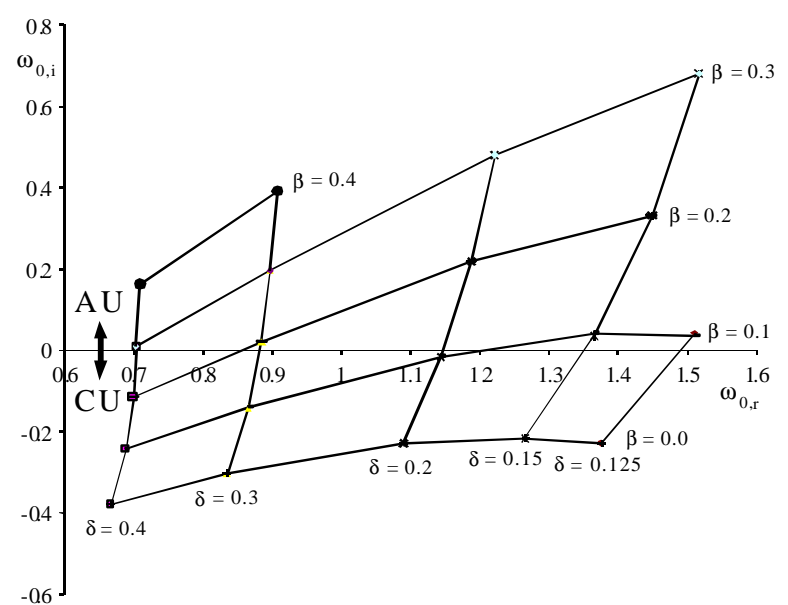

Figure 2 Absolute mode frequencies for the family of velocity profiles shown in equation (1), for different values of the shear layer thickness $\delta$ and the backflow $\beta$.

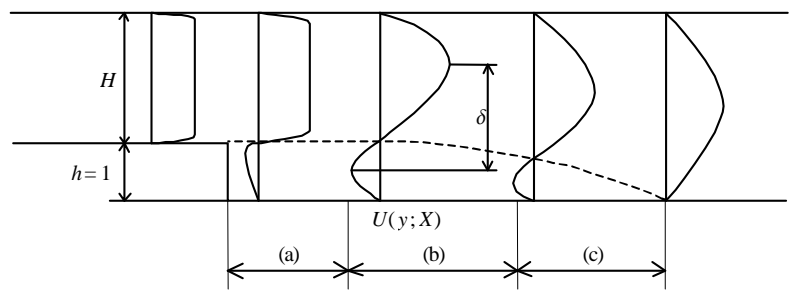

Figure 3. Schematic illustration of different regions in recirculation zone: (a) $\delta<O(1)$ with weak backflow: local absolute frequency $\omega_{0} \sim 1 / \delta$ and locally absolutely stable. (b) $\delta$ $=O(1)$ with weak backflow: local absolute frequency $\mathrm{St} \sim 0.1$ and locally absolutely unstable. (c) $\delta=O(1)$ with weak backflow: local absolute frequency $\mathrm{St} \sim 0.1$ and locally absolutely stable.

due to an absolutely unstable mode in the middle of the recirculation zone persist. With a sufficiently large region of absolutely instability, corresponding to a zone with sufficient backflow, the flow shows a behavior of an unstable oscillator. In this sense, the absolutely unstable section acts as a wave maker for the whole flow, and the frequency of the oscillation is given by $\mathrm{St} \cong 0.1{ }^{4}$ It should be noted that the experimentally observed frequency $96 \mathrm{~Hz}$ in [13] corresponds to $\mathrm{St}=$ 0.092 and is within this range.

To support the conclusions of this analysis regarding the source of sustained oscillations in the separating shear layer, a numerically obtained mean velocity profile for a backward-facing step flow is examined. Simulations are obtained using a two dimensional vortex code, running at Reynolds number of 5000. Results of this simulation, shown in terms of the streamlines close to the steps plotted over a cycle of large-scale vortex shedding within the recirculation zone, are depicted in Figure 4. These simulations show

\footnotetext{
${ }^{4}$ This qualitative observation can be justified by the global mode frequency selection criterion given in [19].
} 
two vortex shedding phenomena, one large eddy which forms in the middle of the recirculation zone over a period of 1.0 (non dimensionalized with respect to the incoming flow velocity and the step height), and another smaller eddy that forms close to the step within a fraction of this time. The corresponding average streamlines which are used in the stability analysis are shown in Figure 5, depicting the primary recirculation zone extending almost six step heights downstream, and a secondary counter recirculation zone closer to the step. These results were obtained without forcing, except for the perturbations introduced due to numerical truncation errors and the statistical noise associated with the random walk simulation of diffusion. Similar results are obtained using finer numerical discretization indicating that it is unlikely that "numerical forcing" is the origin of the unsteadiness. Moreover similar unsteadiness has been observed in other simulations of the same flow, using different numerical methods both in two and three dimensional flows [23-26]. The persistence of these unsteady features and the fact they are observed numerically and experimentally support the claim that they arise due to some intrinsic dynamics, which may have its origin in the instability characteristics of the flow.

Results of the linear stability analysis of this flow are shown in Figure 6. For the purpose of the analysis, the mean velocity profiles corresponding to the average streamlines shown in Figure 5 have been calculated at about 30 sections across the recirculation zone and fitted to a mean velocity profile similar to Eq. (16), and the cusp-map method was used to analyze these profiles and determine the frequency of the absolute mode. As shown in Figure 6, the local absolute mode frequency shows a maximum growth rate near the point $X=1.5$, i.e. close to the middle of the recirculation zone, as predicted before, and the frequency of the mode shows weak dependence on the location in the neighborhood of that point. In the numerical simulation, the formation of a large vortex occurs actively at the middle of the recirculation zone, which corresponds to the location of the maximum growth rate point. The corresponding Strouhal number is $\mathrm{St}=0.083$, also within the range of $\mathrm{St} \cong 0.1$. This value is verified using the numerical simulation results. The Fourier transform of the data is analyzed at different points downstream the step, and the results for the most unstable mode, i.e. the mode with the largest amplitude locally, is shown in Figure 7. The locally dominant mode frequency decreases downstream, as shown in several experiments, and reaches a plateau towards the end of the reciruclation zone at values of $\mathrm{St} \sim 0.08$.
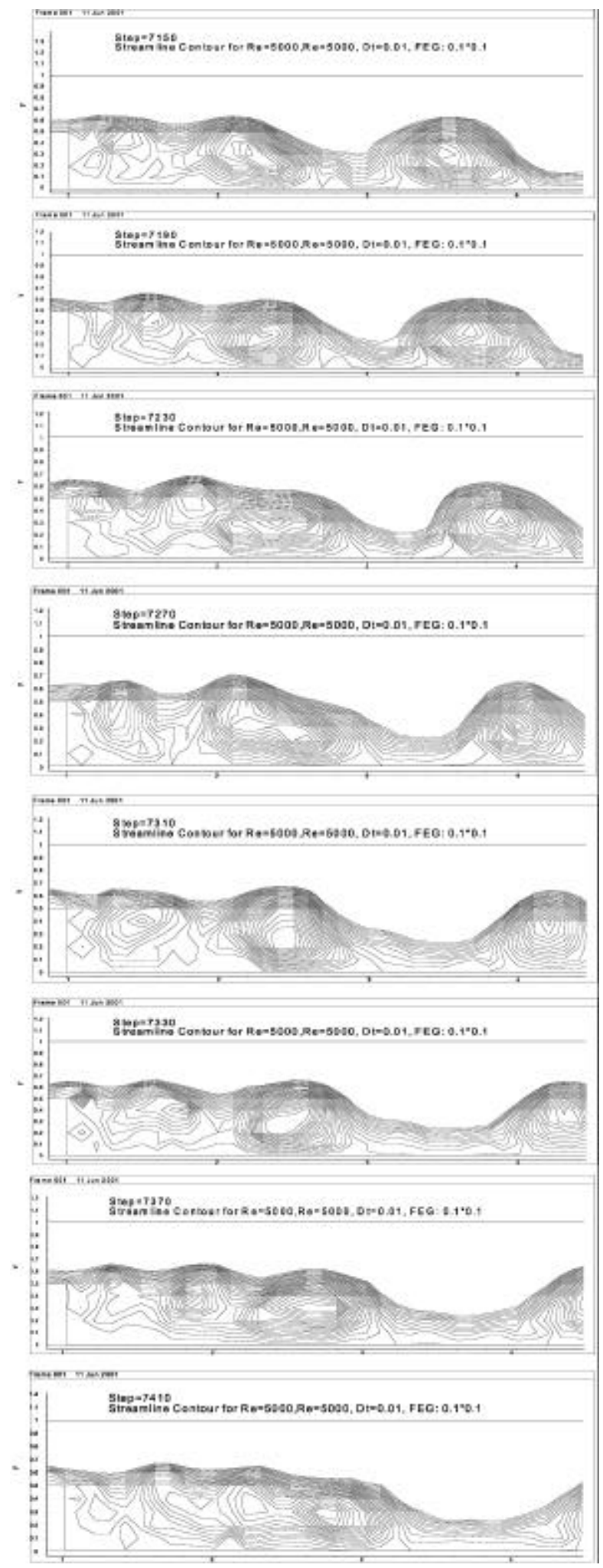

Figure 4 Numerical simulations of the nonreacting flow over a step, Reynolds number 5000, showing the streamlines below a certain value to identify the dynamics of the vortical structures. Note the shedding of eddies at the step followed by pairing. 


\section{II.4 Shear Layer Instability in Reacting Flows}

Flows we have examined so far are isothermal, i.e. with uniform density. When combustion occurs, it changes the shear layer characteristics by introducing a temperature gradient, which in turn gives rise to a density gradient, and affecting the mean velocity profile. To see the effect of the temperature gradient on the stability characteristics, we use the inviscid compressible Rayleigh equation, which is given in $[21,27]$. At the subsonic limit:

$$
\frac{d}{d y}\left[\frac{(U(y)-c)-U^{\prime}(y)}{\Theta(y)} \frac{d \tilde{v}}{d y}\right]=\frac{\alpha^{2}}{\Theta(y)}(U(y)-c) \tilde{v}
$$

where $\Theta$ is the normalized mean temperature profile. field.

Now, we assume the following mean temperature

$$
\Theta(y)=\frac{1+\gamma}{2}+\frac{1-\gamma}{2} \tanh \left(\frac{y}{\delta}\right),
$$

where $\gamma$ is the temperature ratio, which represents a premixed combustion in shear layer. We assume that the thermal boundary layer thickness is of the same order of magnitude as the shear layer thickness. This choice mimics the case where the Prandtl number is near unity.

Keeping $a, b$ and $\delta_{w}$ same as before, the absolute modes for various values of $\gamma, \beta$ and $\delta$ is computed. The result is shown in Figure 8, 9 and 10. For frequency and growth rate, the values obtained are divided by $\int_{y=a}^{b} \frac{U(y)}{\Theta(y)} d y$ for normalization before plotting. As $\gamma$ increases, the flow becomes "less absolutely unstable", i.e. combustion stabilizes the flow by inducing a temperature gradient across the shear zone. Moreover, the flow may switch to a convectively unstable mode at some critical value of $\gamma$. The absolute mode frequency $\omega_{0, r}$ shows weak dependency on the backflow parameter $\beta$ as in the isothermal case. Previous studies of shear layer stability have indeed shown that the important parameters here is the density ratio, and simulations have been used to explain these effects [28]. In particular, the phase speed of the most unstable mode and the convective speed of eddies strongly depends on the density ratio, which suggests that the absolute mode characteristics determined in terms of the group velocity should also show a strong dependency on the density ratio.

It should be noted that the average locations of shear layer and the flame zone may not coincide. Instead a finite offset between the centerline of both

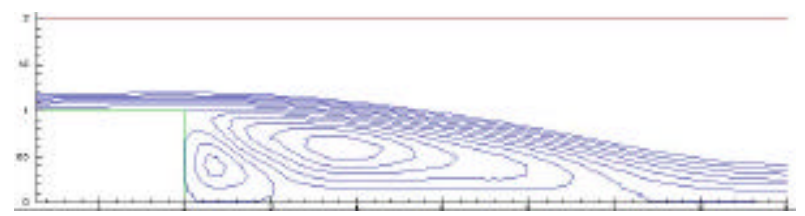

Figure 5 Time-averaged streamlines at $\mathrm{Re}=\mathbf{5 0 0 0}$ without forcing

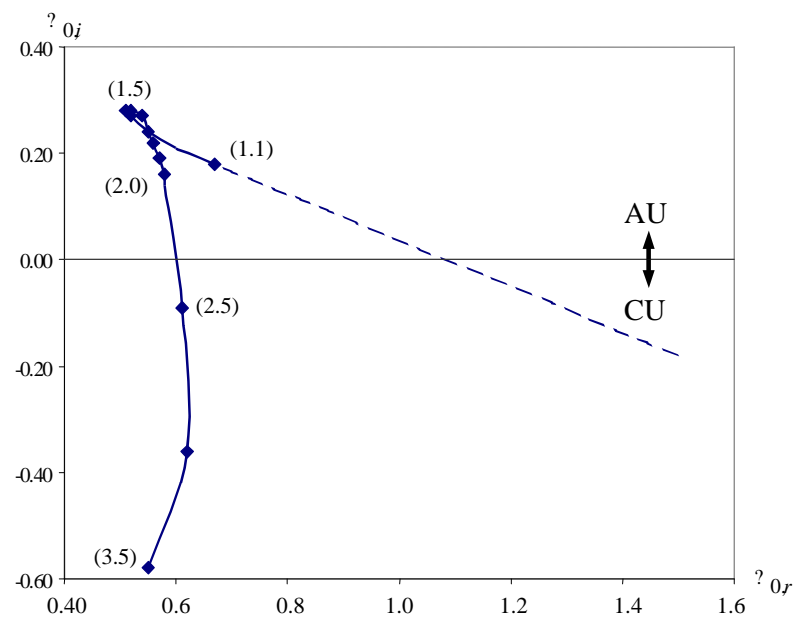

Figure 6 Local absolute mode frequencies for the mean velocity profiles obtained from the numerical simulation of a backward facing step flow shown in Figure 5. In the parenthesis next to the data point, the normalized location of the section $(x / H)$ is shown.

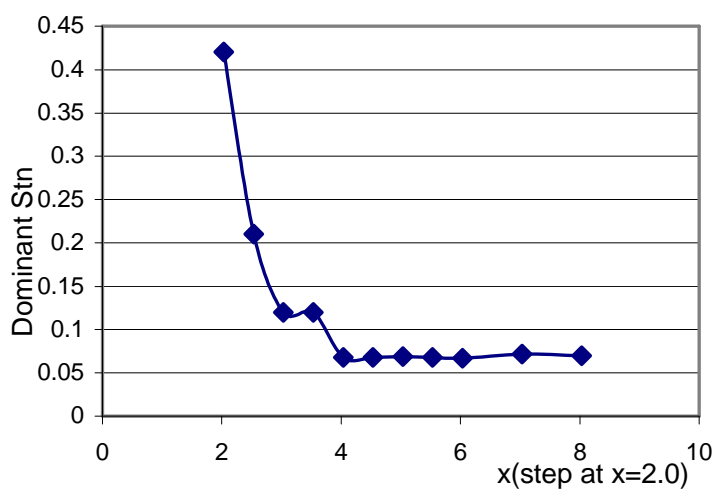

Figure 7 Strouhal number distribution of dominant oscillation at $\mathbf{R e}=5000$ without forcing.

may exit, supported by the fact that the flame starts at the separation point and ends at the upper wall, as shown in [14], while the shear layer starts at the same point but moves downwards towards the lower wall. This offset tends to become larger at higher equivalence ratio as the burning velocity of the premixed flame increase forcing the flame to move towards the outer edges of the large eddies instead of burning closer to their centers. To study the impact of the offset, we replace $y$ with $y-\Delta$ in Eq. (18). The impact of the offset is shown in Figure 11. At small temperature 


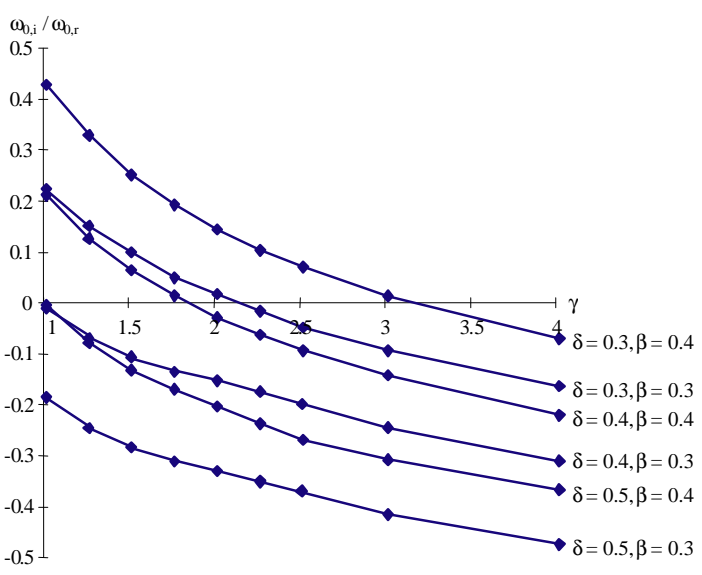

Figure 8 Relative growth rate $\omega_{0, i} / \omega_{0, r}$ of absolute mode for various values of $\gamma, \delta$, and $\beta$.

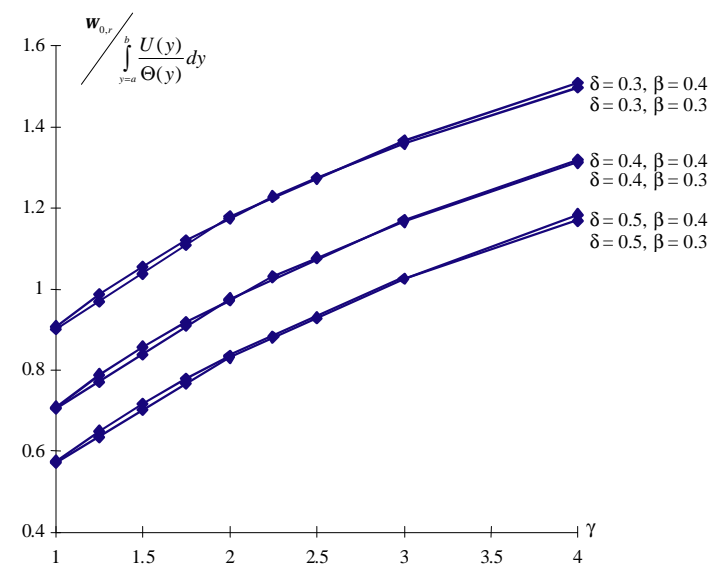

Figure 9 Normalized frequency of absolute mode for various values of $\gamma, \delta$, and $\beta$.

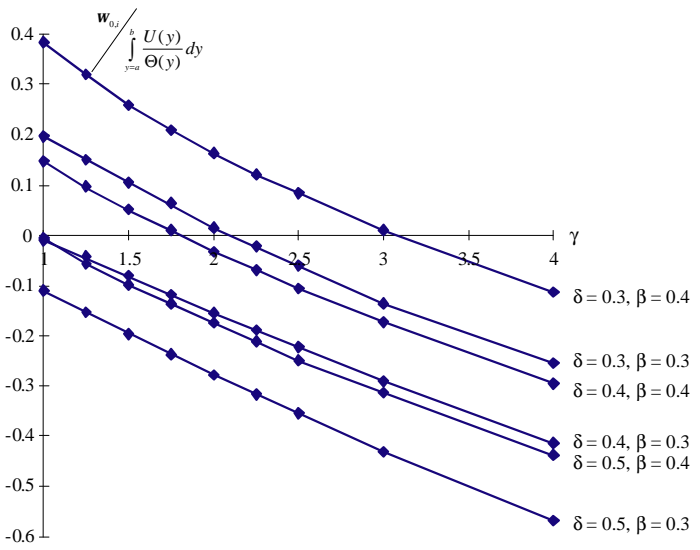

Figure 10 Normalized growth rate of absolute mode for various values of $\gamma, \delta$, and $\beta$.

ratios, i.e. at conditions of lean combustion, the offset is expected to be very small. In any case, results show that, under these conditions, the offset has almost no impact of the mode frequency and growth rate. At higher temperature ratio, as the equivalence ratio moves towards stoichiometry, the offset is expected to be finite and results show that its impact becomes important: at small offset, the flow is convectively unstable, but at larger values, it becomes absolutely unstable. This is clearly shown in Figure 11 where the curve corresponding to $\omega_{0, i} / \omega_{0, r}=0$ delineates the border between the absolutely unstable and the convectively unstable regimes. It should be added here that the offset and the temperature ratio are not totally independent since, for the same fuel, they both depend on the equivalence ratio; in fact, it can be stated that they both increase as the fuel concentration in the mixture approaches stoichiometry. This is shown schematically by the thick arrow in Figure 11, where a line is drawn to connect qualitatively the possible trajectory of states of the flow as the equivalence ratio is increased from lean conditions to chemically stoichiometric conditions.

The second effect of combustion, that is changing the mean velocity profile due to the exothermic effects, is harder to identify. In general, there is a tendency for combustion to reduce the reattachment length and increase the velocity within the recirculation zone, with an associated rise in backflow, these effects are currently under investigation.

In summary, the results of linear stability analysis of shear flow show the following characteristics:

1) The shear layer instability can produce self-sustained oscillations. This is due to the presence of absolutely unstable modes which arise when the mean velocity profile shows a strong back-flow at sections where the shear layer thickness is relatively small.

2) The absolutely unstable modes in a backward-facing step flow show an oscillation frequency close to $\mathrm{St} \approx 0.1$. The apparent universality of this value is explained by its dependence on the thickness of the layer, about a step height, and the value of backflow. Also, the predicted frequency matches the experimental observation (of $\mathrm{St}=0.092$ ) in [13].

3) The "global" separating shear layer mode frequency is the same as the local most absolutely unstable mode, i.e. the absolutely unstable mode with the highest growth rate within the separation zone.

4) The impact of these modes is most pronounced at the middle of the recirculation zone, while closer to the step small scale shedding due to other, most likely convective modes, is observed.

5) Large temperature gradients across the shear layer force the flow to become more absolutely stable, and hence stability characteristics can be affected by combustion. Flows at higher equivalence ratio can become absolutely unstable due to the offset between the shear layer and the flame boundary. 


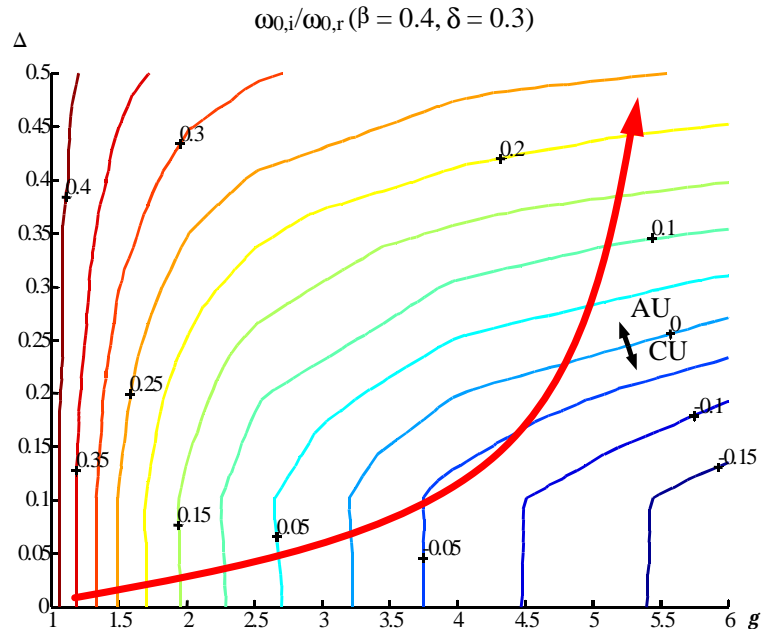

Figure 11. The impact of the temperature distribution on the properties of the absolute instability, shown in terms of the ratio of absolute growth rate to absolute frequency $\left(\omega_{0, j} / \omega_{0, r}\right)$ for various values of temperature ratio $\gamma$ and offset $\Delta$ with $\delta=0.3$ and $\beta=0.4$. The thick arrowed line shows a possible path for increasing the equivalence ratio.

\section{Acoustic Gain from Shear Layer Driven Heat Release}

As mentioned in the introduction, combustion instability is sometimes exhibited at multiple frequencies, not all of which can be attributed to acoustic frequencies. The hypothesis that is put forth in this paper is that some of these frequencies could be due to an absolutely unstable shear-layer mode. The analysis conducted in the previous section showed that a backward step configuration can lead to shear layer structures that generate self-sustained oscillations with a Strouhal number $\mathrm{St}=0.1$. The question is then whether oscillations at this frequency can lead to significant pressure oscillations. In this section, a preliminary analysis is carried out and compared with the experimentally observed pressure amplitudes in [13].

The acoustic field is approximated using a Galerkin approximation $[29,30]$, in which the unsteady pressure $p^{\prime}$ is expressed as:

$$
p^{\prime}(x, t)=\bar{p} \sum_{i=1}^{n} \psi_{i}(x) \zeta_{i}(t),
$$

where $\psi_{i}(x)$ and $\zeta_{i}(t)$ are modal shape and amplitude. Assuming that the heat release is localized at $x=x_{f}$, the amplitude, $\zeta_{i}(t)$, can be shown to be governed by (see Ref. [31])

$$
\frac{d^{2} \zeta_{i}}{d t^{2}}+\Omega_{i}^{2} \zeta_{i}=\gamma a_{o} E^{-1} \psi_{i}\left(x_{f}\right) \ddot{q}_{f}^{\prime},
$$

where $\Omega_{i}$ is the acoustic frequency, $a_{o}=(\gamma-1) / \gamma \bar{p}$, $E_{i}=\int_{0}^{L} \psi_{i}(x)^{2} d x$, and $q_{f}^{\prime}$ is the heat release rate per unit area at $x_{f}$. Using Laplace transforms of Eq. (19) and Eq. (20), and noting that the configuration in [13] had two dominant acoustic modes, we get

$$
p^{\prime}(x)=(\gamma-1) \sum_{i=1}^{2} \psi_{i}(x) \psi_{i}\left(x_{f}\right) \frac{s}{E_{i}\left(s^{2}+\Omega_{i}^{2}\right)} q_{f}^{\prime}
$$

It is worth noting at any frequency, $\omega$, including a shear-layer absolute frequency, $p$ ' can be evaluated simply by setting $s=j \omega$. In Ref. [13], $\Omega_{1}=302 \mathrm{rad} / \mathrm{s}$ and $\Omega_{2}=779 \mathrm{rad} / \mathrm{s}$, which are the quarter and the three quarter modes, $x_{f}=1.1 \mathrm{~m}, x_{s}=1.4 \mathrm{~m}, L=2.4 \mathrm{~m}$ and $\gamma=1.4$. As shown in the previous section, this configuration may be capable of generating a hydrodynamic instability with $\mathrm{St} \cong 0.1$, which corresponds to a frequency of $\omega=603 \mathrm{rad} / \mathrm{s}$. The amount of heat release rate perturbation $q^{\prime}$ in a typical backward facing step flow due to shear layer perturbations has been observed to be $15 \%$ in an experimental investigation in [32] and $25 \%$ in a numerical study in [33] of the mean heat release rate, $\bar{q}$. Using a value of $q_{f}^{\prime}=0.15 \bar{q}$, the pressure amplitude at $\omega=603 \mathrm{rad} / \mathrm{s}$ can be calculated using Eq. (21), which resulted in $p^{\prime}=0.08$ psi. This matches the experimentally observed value of $0.07 \mathrm{psi}$ at the same $\omega$.

\section{Reduced Order Modeling Using POD Analysis}

In the preceding sections, we have discussed the dynamics of shear layers in the context of linear stability analysis, and showed that it can generate selfsustained oscillations under certain conditions. An approximate estimation of the amplitude of pressure oscillation due to the shear layer dynamics is given in the previous section, which supports the conclusion that self-sustained oscillations due to the absolute mode of the shear layers can cause thermoacoustic instability. These discussions demonstrate that an accurate model of thermoacoustic instability must encompass the three components, acoustics, heat release dynamics, and shear layer dynamics.

The analytical tool used in section II to model shear-layer instability is linear stability theory. While the theory may be used to gain insight into stability characteristics of shear flow and their possible role in "post transition states", and help explain and generalize results from numerical simulations, a quantitative behavior of shear layers cannot easily be obtained from linear stability analysis. While detailed numerical 
simulations can be accurate enough, the huge computational time needed for detailed simulations makes them insufficient for control applications. What is more useful is a reduced-order model for shear layer dynamics, that is capable of utilizing numerical results available, and leads towards a more complete quantitative model of the overall combustion dynamics. In this section, we discuss such a tool, POD analysis, and use numerical results to obtain a reduced order model of the flow field in the presence of hydrodynamic instability.

The POD is a tool for extracting coherent structures from numerical data, and it is a systematic and optimal way to derive reduced-order models. In POD, one uses numerical results to construct a space of optimal basis functions that describe the different modes of the flow, and apply these functions to construct time-dependent ordinary differential equations (ODEs) that determine the amplitudes of the corresponding modes under different conditions. The eigenvalues and eigenfunctions are obtained from the covariance matrix of the data evaluated at different time steps, while the ODEs are obtained from a Galerkin expansion of the dependent variables in these basis functions, and projecting the original Navier-Stokes equations onto their space [34].

As mentioned before, the numerical data are obtained from the two-dimensional simulation of a separating flow downstream of a backward-facing step using a vortex code. This code has been described extensively in the literature and several simulations of similar flow have been used to demonstrate its validity $[17,35]$. The code was applied to the case of $1: 2$ expansion, similar to the experiment in [13] with $\mathrm{Re}=5000$. For the backward facing step flow, detailed formulation of the equations for POD modeling has been developed in [36]. We apply the same procedure as in [36] here, but use a different numerical data obtained from a vortex code as mentioned above. The objectives of applying this procedure here are to: (1) confirm that the dominant mode in these data is that whose frequency is $\mathrm{St} \cong 0.1$, and (2) examine the accuracy of a reduced model of this complex flow.

\section{IV.1 Eigenvalues and Basis Functions}

Using 100 snapshots for the flow with a time step of $0.1,100$ eigenvalues and basis functions are obtained with the following procedures [36]:

1. The results are organized in the form $u\left(\bar{x}, t_{k}\right)$, where $u$ is the velocity, $\bar{x}$ is the spatial and $t$ is the time coordinate at $N(=100)$ different time steps, $t_{k}$, $(k=1, \ldots, N)$
2. The velocity, $u\left(\bar{x}, t_{k}\right)$, is decomposed such that $u(\bar{x}, t)=u_{m}(\bar{x})+u^{\prime}(\bar{x}, t)$, where $u_{m}$ is the mean velocity and $u^{\prime}$ is the perturbation.

3. The covariance matrix $C$, which is defined as $C_{i j}=\frac{1}{N} \int_{\Omega} u^{\prime}\left(\bar{x}, t_{i}\right) u^{\prime}\left(\bar{x}, t_{j}\right) d \Omega$, is computed.

4. The eigenvalue problem, $C W=\lambda W$, is solved to obtain the eigenvalues $\lambda_{i}$.

5. The basis functions, $\Phi_{i}$, are constructed such that:

$$
\Phi_{i}=\sum_{k=1}^{N} W_{i k} u\left(\bar{x}, t_{k}\right) \text {. }
$$

The resulting eigenvalues are show in Figure 12. Each eigenvalue corresponds to the energy contained in each mode, i.e. $\lambda_{i}=\frac{1}{N} \sum_{j=1}^{N}\left(\Phi_{i}, u^{\prime}\left(\bar{x}, t_{j}\right)\right)^{2}$ where $(\cdot, \cdot)$ denotes inner product. As can be seen from the figure, the energy contained in the first 10 modes correspond $96 \%$ of the total energy, showing that the first few modes may be sufficient to describe the flow accurately. We can expect that the accuracy of the reduced order model should improve as the number of modes used to construct the model increases. In the following we examine this statement for the backward facing step flow.

\section{IV.2 Reduced Order Modeling}

Using the basis functions obtained in the previous section, a reduced order model is developed by projecting the NS equations onto the basis functions using a Galerkin expansion. Detailed formulation of the equations in the backward facing step is summarized in [36]. The reduced NS equation determines the time evolution, $\alpha_{i}(t)$, of the each modes, and the resulting velocity profile, $u$, as follows;

$$
u(\bar{x}, t) \cong u_{m}(\bar{x})+\sum_{i=1}^{N^{*}} \alpha_{i}(t) \Phi_{i}
$$

where $u_{m}$ is the mean velocity profile and $N^{*}$ is the number of the modes used to construct the reduced order model. The coefficients $\alpha_{i}$ are obtained by numerically integrating the following nonlinear ODE:

$$
\dot{\alpha}=A \alpha+\alpha^{T} M \alpha+e
$$

where

$$
\begin{aligned}
& A_{i j}=-\left(\Phi_{j} \cdot \nabla u_{m}, \Phi_{i}\right)-\left(u_{m} \cdot \nabla \Phi_{j}, \Phi_{i}\right)-\frac{1}{\operatorname{Re}}\left(\nabla \Phi_{j}, \nabla \Phi_{i}\right), \\
& M_{i k l}=-\left(\Phi_{k} \cdot \nabla \Phi_{l}, \Phi_{i}\right), \text { and } e_{i}=-\left(u_{m} \cdot \nabla u_{m}, \Phi_{i}\right) .
\end{aligned}
$$

The initial condition for the ODE is obtained by projecting the original initial condition to the POD basis functions, i.e. $\alpha(0)=(u(\bar{x}, 0), \Phi)$. 
With $N^{*}=10$, the coefficients of the nonlinear ODE, $A, M$ and $e$ are computed. An analysis of the eigenvalues of $A$ shows that the frequency and damping ratio of the first two POD modes in Figure 12 are $\mathrm{St}=0.083$ and -0.097 , respectively. Similarly, the frequency and damping ratio for the third and fourth mode shown in Figure 12 are 0.23 and -0.0382 , respectively. It can be seen that the first of the two frequencies with $\mathrm{St}=0.083$ has a larger growth rate, and hence corresponds to the most unstable mode, which is consistent with the result obtained from the linear stability analysis carried out in section II. Obviously, due to the assumption that the underlying flow contains only one dominant mode, the results in section II yielded only the most dominant Strouhal number. Equation (22) represents the flow field as the combination of the basis functions, $\Phi_{i}$, and equation (23) determines the coefficients of these functions in time. Therefore, these two equations completely describe the POD reduced order model, and it is possible to simulate the flow field.

Figure 13 shows a comparison between the actual data and the POD reconstruction using 4, 6 and 10 modes, and show that the reduced order model in (22) and (23) is reasonably accurate. The model behavior at two different points in the flow is presented in the figure, and corresponds to the anti-node and the node of the first mode. Clearly the reconstruction accuracy depends on the dominant dynamics at the location and the number of modes, the more significant the higher frequencies are, the larger the number of modes that should be used in the reconstruction.

\section{Summary}

In this paper, the possibility that a hydrodynamic mechanism may introduce self-sustained oscillations in a combustor is examined. Motivated by several observations in the literature where combustion instability is reported to occur at multiple frequencies, conditions under which unstable hydrodynamic modes can be present and cause combustion instability are investigated. Using linear stability analysis of separating shear layers, it is shown that under conditions most often encountered in non reacting and reacting flows downstream a backward facing step, the flow possesses instability modes which may lead to self-sustained hydrodynamic oscillations. The associated frequencies are determined by the shear flow properties, e.g., the shear layer thickness, the amount of backflow, the temperature or density across the shear zone, and the offset between the shear and burning zones. The analysis is used to explain results of numerical simulations of non reacting flows which

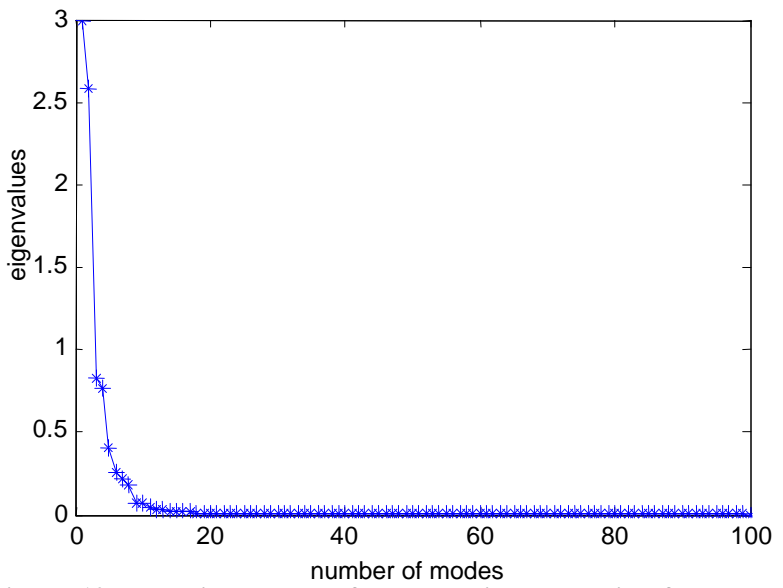

Figure 12 The eigenvalues of the covariance matrix of the data set obtained from the numerical simulation of a backward facing step flow $\mathrm{Re}=\mathbf{5 0 0 0}$.

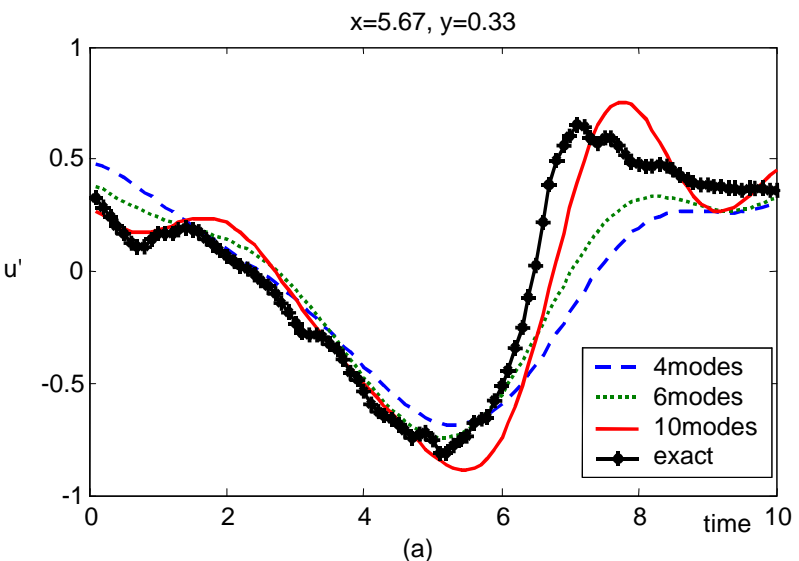

(a)

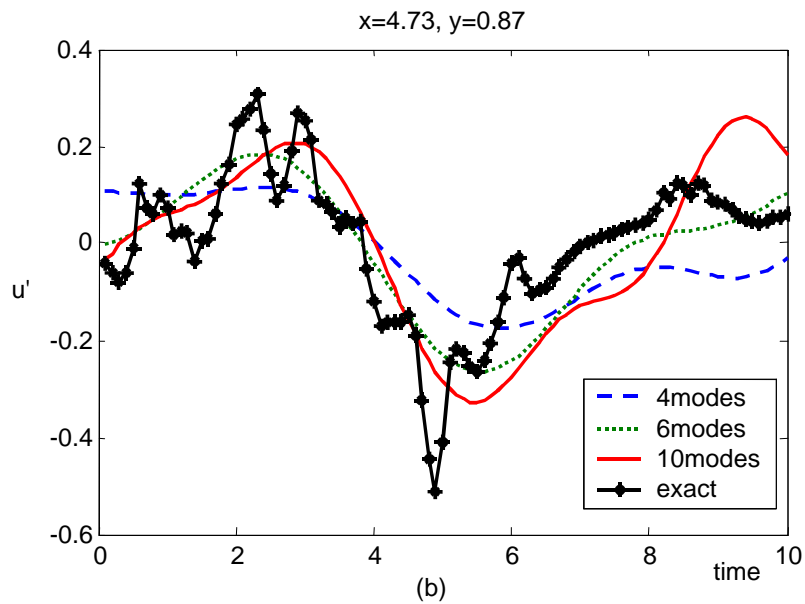

Figure 13 Comparison between data and POD model for u' at (a) an anti-node of the first mode located at $(5.67,0.33)$ and (b) a node of the same mode, $(4.73,0.87)$. The POD model uses 4, 6 and 10 modes to reconstruct the data. 
exhibit strong unsteadiness at frequencies close to those predicted for certain modes, and is shown to match pressure amplitudes observed in experimental investigations of a backward-facing step combustor in [13]. In order to determine an overall combustion dynamics model that accurately represents the effect of shear-layer instability so as to carry out a model-based control design, POD analysis of the numerical data is carried out, and is shown that the first few POD modes are capable of capturing more $90 \%$ of the energy in the flow. While the main focus of the paper has been on combustors with a backward-facing step, the tools introduced here are applicable for more general configurations.

\section{Acknowledgments}

This work is sponsored by the National Science Foundation, grant no. ECS 9713415, and the Office of Naval Research, grant no. N00014-99-1-0448, and the Air Force Office of Sponsored Research, F49620-00-C0038 .

\section{$\underline{\text { References }}$}

[1] B.T. Zinn, Pulsating Combustion. Advanced Combustion Methods. Academic Press, 1986.

[2] F. Culick, "Combustion Instabilities in LiquidFueled Propulsion Systems," AGARD Conference Proceedings, Paper 1, 450, 1988.

[3] M. Fleifil, A.M. Annaswamy, Z.A. Ghoneim and A.F. Ghoniem, "Response of a Laminar Premixed Flame to Flow Oscillations: A Kinematic Model and Thermoacoustic Instability Results," Combustion and Flame, vol. 106, issue 4, pp.487-510, 1996.

[4] A.M. Annaswamy, M. Fleifil, J.P. Hathout and A.F. Ghoniem, "Impact of Linear Coupling on the Design of Active Controllers for the Thermoacoustic Instability," Combust. Sci. and Tech., vol. 128, pp.131-180, 1997.

[5] T. Lieuwen, Y. Neumeier and B. T. Zinn., "The Role of Unmixedness and Chemical Kinetics in Driving Combustion Instabilities in Lean Premixed Combustors," Combust. Sci. and Tech., vol. 135, pp.193-211, 1998.

[6] A.P. Dowling, "A Kinematic Model of a Ducted Flame,” J. Fluid Mech., vol. 394, pp51-72, 1999.
[7] S. Park, A.M. Annaswamy and A.F. Ghoniem, "Heat Release Dynamics Modeling for Combustion Instability Analysis of Kinetically Controlled Burning," AIAA 2001-0780, 39 ${ }^{\text {th }}$ Aerospace Sciences Meeting and Exhibit, 2001.

[8] J.O. Keller, L. Vaneveld, D. Korschelt, G.L. Hubbard, A.F. Ghoniem, J.W. Daily, and A.K. Oppenheim, "Mechanism of Instabilities in Turbulent Combustion Leading to Flashback," AIAA J., vol. 20, pp.254-262, 1982.

[9] S.R.N. DeZilwa, J.H. Uhm and J.H. Whitelaw, "Combustion Oscillations Close to the Lean Flammability Limit," Combust. Sci. and Tech, vol. 160, pp.231-258, 2000.

[10] K.C. Schadow, E. Gutmark, T.P. Parr, D.M. Parr, K.J. Wilson, and J.E. Crump, "Large-Scale Coherent Structures as Drivers of Combustion Instability," Combust. Sci. and Tech., vol. 64, pp.167-186, 1989.

[11] C.O. Paschereit, W. Weisenstein and E. Gutmark, "Role of Coherent Structures in Acoustic Combustion Control," AIAA 98-2433, 29th AIAA Fluid Dynamics Conference, 1998.

[12] C.O. Paschereit, E. Gutmark and W. Weisenstein, "Reduction of Pressure Oscillations by Direct Excitation of Gas-Turbine Burner's Shear Layer," AIAA 2000-1028, 38 ${ }^{\text {th }}$ Aerospace Sciences Meeting and Exhibit, 2000.

[13] J.M. Cohen and T.J. Anderson, "Experimental Investigation of Near-Blowout Instabilities in a Lean, Premixed Step Combustor," AIAA 96-0819, 34 ${ }^{\text {th }}$ Aerospace Sciences Meeting \& Exhibit, 1996.

[14] R.W. Pitz and J.W. Daily, "Combustion in a Turbulent Mixing Layer Formed at a RearwardFacing Step,” AIAA J., vol. 21, pp.1565-1570, 1983.

[15] D.M. Driver, H. Lee Seegmiller and J.G. Marvin, "Time-Dependent Behavior of a Reattaching Shear Layer,” AIAA J., vol. 25, pp.914-919, 1983.

[16] A.F. Heenan and J.F. Morrison, "Passive Control of Backstep Flow," Experimental Thermal and Fluid Science, vol. 16, pp.122-132, 1988.

[17] H.N. Najm and A.F. Ghoniem, "Numerical Simulation of the Convective Instability in a Dump," AIAA J., vol. 29, pp.911-919, 1991. 
[18] P. Huerre and P.A. Monkewitz, "Absolute and Convective Instabilities in Free Shear Layers," J. Fluid Mech., vol. 159, pp.151-168, 1985.

[19] P. Huerre and P.A. Monkewitz, "Local and Global Instabilities in Spatially Developing Flows," Ann. Rev. Fluid Mech., vol. 22, pp.473-537, 1990.

[20] P.G. Drazin and W.H. Reid, Hydrodynamic Stability, Cambridge University Press, 1981.

[21] P.J. Schmid and D.S. Henningson, Stability and Transition in Shear Flows, Springer, 2001.

[22] K. Kupfer, A. Bers, and A.K. Ram, "The Cusp Map in the Complex-Frequency Plane for Absolute Instabilities," Phys. Fluids, vol. 30, pp.3075-3082, 1987.

[23] C. Fureby, "Large Eddy Simulation of RearwardFacing Step Flow," AIAA J., vol. 37, pp.1401-1410, 1999.

[24] H. Le, P. Moin and J. Kim, "Direct Numerical Simulation of Turbulent Flow over a BackwardFacing Step," J. Fluid Mech., vol. 330, pp.349-374, 1997.

[25] L. Kaiktsis, G.E. Karniadakis and S.A. Orszag, "Unsteadiness and Convective Instabilities in TwoDimensional Flow over a Backward-Facing Step," J. Fluid Mech. vol. 321, pp.157-187, 1996.

[26] L. Kaiktsis, G.E. Karniadakis and S.A. Orszag, "Onset of 3-Dimensionality, Equilibria, and Early Transition in Flow over a Backward-Facing Step," J. Fluid Mech., vol. 231, pp. 501-528, 1991.

[27] L. Lees and C.C. Lin, "Investigation of the Stability of the Laminar Boundary Layer in a Compressible Fluid,” NACA TN 1115, 1946.

[28] M.C. Soteriou and A.F. Ghoniem, "Effects of the Free-Stream Density Ratio on Free and Forced Spatially Developing Shear Layers," Phys. Fluids, vol. 7, pp. 2036-2051, 1995.

[29] B. T. Zinn and M. Lores, "Application of The Galerkin Method in the Solution of Nonlinear Axial Combustion Instability Problems in Liquid Rockets," Combust. Sci. and Tech, vol. 4, pp. 269-278, 1972.

[30] F. Culick, "Nonlinear behavior of acoustic waves in combustion chambers", Acta Astronautica, vol. 3, pp.715-756, 1976.
[31] A.M. Annaswamy, M. Fleifil, J.P. Hathout, and A.F. Ghoniem, "Impact of Linear Coupling on the Design of Active Controllers for The Thermoacoustic Instability", Combust. Sci. and Tech., vol. 128, pp.131-180, 1997.

[32] K. R. McManus, U. Vandsburger and C. T. Bowman, "Combustor Peroformance Enhancement through Direct Shear Layer Excitation," Combust. Sci. and Tech., vol. 82, pp.75-92, 1990.

[33] H. Najm and A. F. Ghoniem, "Modeling Pulsating Combustion Due to Flow-Flame Interactions in Vortex-Stabilized Pre-Mixed Flames," Combust. Sci. and Tech., vol. 94, pp.259-278, 1993.

[34] P. Holmes, L. Lumley and G. Berkooz, Turbulence, Coherent Structures, Dynamical Systems and Symmetry, Cambridge University Press, 1996.

[35] J. Sethian and A.F. Ghoniem, "Validation of the Votex Method," J. Comput. Phys., vol. 74, pp.283317, 1988.

[36] S.S. Ravindran, "Reduced-Order Adaptive Controllers for Fluid Flows Using POD," Journal of Scientific Computing, vol. 15, pp.457-478, 2000. 